International Journal of Applied Mathematics

Volume 29 No. 2 2016, 263-270

ISSN: 1311-1728 (printed version); ISSN: 1314-8060 (on-line version)

doi: http://dx.doi.org/10.12732/ijam.v29i2.9

\title{
DYNAMICAL ANALYSIS OF \\ AN AXIALLY VIBRATING NANOROD
}

\author{
Hanif Heidari \\ School of Mathematics and Computer Sciences \\ Damghan University \\ Damghan, P.O. Box 36715-364, IRAN
}

\begin{abstract}
The aim of this paper is to study dynamical properties of an axially vibrating uniform nanorod. This analysis is performed by describing the model as an infinite-dimensional state-space system, with bounded control operator. In the absence of control forces, the system with homogeneous boundary conditions generates a strongly continuous semigroup. It is proved that the associated eigenfunctions form a Riesz basis for the energy space. It is shown that axial vibration of nanorod is stable phenomena but not exponentially stable. A necessary and sufficient condition for the approximate controllability is given.
\end{abstract}

AMS Subject Classification: 34H05, 93B05, 35Q74

Key Words: nanorod, vibration, stability, controllability

\section{Introduction}

With the rapid development of nanotechnology, miniaturized structures with nanoscale features can be precisely designed, manufactured and applied in the so-called Nano-Electro-Mechanical Systems (NEMS) [9, 7]. Since, Carbon Nano Tubes (CNTs) and nonlocal elastic beams with nanoscale thickness (nanorod) have good electrical properties and high mechanical strength, they are most popular in NEMS [11, 12]. Moreover, they are of significance for use in advanced catalysts, adsorbents, composite materials and ceramics [2].

$\begin{array}{ll}\text { Received: January 21, } 2016 & \text { (c) } 2016 \text { Academic Publications }\end{array}$ 
Elastic properties of CNTs can be determined by using axial vibration experiments. Although flexural experiments are used when determining Youngs modulus, axial vibrations can also be used. As stated by Fonoberov and Balandin [6], pure axial vibration mode can also be observed. Axial external forces that may act on nanorods leads to axial vibration of them [1]. Due to the vast applications of nanorods, controllability and stability analysis of an axially vibrating nanorod is crucial for many of their future uses.

The rest of this paper is organized as follows. In Section 2, the axial vibration of the nanorods is described by a fourth order partial differential equation with mixed derivative. The differential equation is formulated as an abstract differential equation in Section 3. The close analytical solution of the equation is achieved by using semigroup theory. It is shown that the infinitesimal generator of the semigroup is a Riesz-spectral operator. Moreover, stability of axial vibration of nanorod is investigated. In Section 4, a necessary and sufficient condition for the approximate controllability is obtained by using the properties of Riesz-spectral operators.

\section{Problem Formulation}

Consider the nanorod in the physical domain determined by a thin elastic rod of length $l$. Let $\Omega=(0, l)$ be a bounded open set in $\mathbb{R}, T>0$ and $D=\Omega \times(0, T)$. The PDE describing the axial vibration of a nanorod over domain $D$ can be expressed as follows $[1,13]$ :

$$
\mathbb{E} \mathbb{A} \frac{\partial^{2}}{\partial x^{2}} u(x, t)+b(x) f(t)=\left(1-\left(e_{0} a\right)^{2} \frac{\partial^{2}}{\partial x^{2}}\right) m \frac{\partial^{2}}{\partial t^{2}} u(x, t)
$$

where $u(x, t)$ is axial displacement, $\mathbb{E}$ is the conventional Young's modulus, $\mathbb{A}$ is the cross-sectional area of the nanorod, $m$ is the mass per unit length, $a$ is an internal characteristic length, $e_{0}$ is a constant, $b(x)$ represents the shaping function around the control point $x_{0}$ and $f(t)$ axially distributed external control force. The equation (1) is obtained by using nonlocal continuum theory of Eringen. For more information regarding the established theory by Eringen, see $[4,5]$. Without loss of generality, $\mathbb{A}=\mathbb{E}=1$ is assumed throughout this paper for simplicity.

The initial and boundary conditions are: 


$$
\begin{aligned}
& u(x, 0)=f_{1}(x) \\
& \frac{\partial}{\partial t} u(x, 0)=f_{2}(x) \\
& u(0, t)=0 \\
& u(l, t)=0,
\end{aligned}
$$

where $f_{1}(x)$ and $f_{2}(x)$ are given real valued functions.

\section{Semigroup Formulation}

The system of equations (1)-(5) can be transformed to an abstract differential equation. As state space we choose the energy space $\mathcal{H}$, which is the Hilbert space $H^{2}(\Omega) \cap H_{0}^{1}(\Omega) \times H^{2}(\Omega) \cap H_{0}^{1}(\Omega)$ with the inner product

$$
\left\langle\left(\begin{array}{l}
u_{1} \\
u_{2}
\end{array}\right),\left(\begin{array}{l}
w_{1} \\
w_{2}
\end{array}\right)\right\rangle_{e}=\frac{m}{2} \int_{0}^{l}\left(e_{0} a\right)^{2} \frac{d^{2} u_{1}}{d x^{2}} \frac{\overline{d^{2} w_{1}}}{d x^{2}}+J\left(u_{2}\right) \overline{J\left(w_{2}\right)} d x,
$$

where $J=m\left(1-\left(e_{0} a\right)^{2} \frac{d^{2}}{d x^{2}}\right)$.

On this state space, it is easy to show that $J$ is an bounded and invertible operator. We write (1)-(5) in the following abstract form:

$$
\left\{\begin{array}{l}
\frac{d}{d t}\left(\begin{array}{c}
u \\
u_{t}
\end{array}\right)=A\left(\begin{array}{c}
u \\
u_{t}
\end{array}\right)+B f \\
\left.\left(\begin{array}{c}
u \\
u_{t}
\end{array}\right)\right|_{t=0}=\left(\begin{array}{l}
f_{1} \\
f_{2}
\end{array}\right),
\end{array}\right.
$$

with $u_{t}=\frac{d u}{d t}, B=\left(\begin{array}{c}0 \\ J^{-1}(b(x))\end{array}\right)$ and $A$ is given by

$$
A\left(\begin{array}{l}
u_{1} \\
u_{2}
\end{array}\right)=\left(\begin{array}{c}
u_{2} \\
J^{-1}\left(\frac{d^{2} u_{1}}{d x^{2}}\right)
\end{array}\right)
$$

where $J^{-1}$ denotes the inverse of the operator $J$ and

$$
D(A)=H^{2}(\Omega) \cap H_{0}^{1}(\Omega) \times H^{2}(\Omega) \cap H_{0}^{1}(\Omega) .
$$

Definition 1. Let $\mathcal{A}$ be a closed linear operator on the Hilbert space, $Z$, with simple eigenvalues $\left\{\gamma_{n}, n \geq 1\right\}$ and its corresponding eigenvalues $\left\{\phi_{n}, n \geq\right.$ $1\}$ form a Riesz-basis in $Z$. If the closure of $\left\{\gamma_{n}, n \geq 1\right\}$ is totally disconnected, then $\mathcal{A}$ is called Riesz-spectral operator. 
The properties of Riesz-spectral operators have been widely studied on mathematical systems theory (for example see [3, 8, 15] and references therein). In the following, we will prove that $A$ is Riesz-spectral operator.

Theorem 2. The operator $A$ as defined in (8) and (9) is the infinitesimal generator of a strongly continuous semigroup on $\mathcal{H}$.

Proof. It is enough to show that the operator $A$ is a Riesz-spectral operator, the result follows from Theorem 2.3.5 in [3]. By using standard arguments (see [3], Theorem A.3.46), it can be shown that the operator $A$ is closed and densely defined on $\mathcal{H}$. Let $\Lambda$ denotes the set of eigenvalues of $A$. It suffices to show that the eigenvalues of $A$ are simple, totally disconnected, satisfy

$$
\sup _{\lambda \in \Lambda} \operatorname{Re}(\lambda)<\infty
$$

and its eigenfunctions form Riesz basis. Thus, we begin by calculating the eigenvalues and eigenfunctions of $A$. From (8) we have that

$$
A\left(\begin{array}{l}
u_{1} \\
u_{2}
\end{array}\right)=\lambda\left(\begin{array}{l}
u_{1} \\
u_{2}
\end{array}\right) \Leftrightarrow\left\{\begin{array}{l}
u_{2}=\lambda u_{1} \\
J^{-1}\left(\frac{d^{2} u_{1}}{d x^{2}}\right)=\lambda u_{2} .
\end{array}\right.
$$

Therefore, $u_{2}=\lambda u_{1}$ and

$$
J^{-1}\left(\frac{d^{2} u_{1}}{d x^{2}}\right)=\lambda^{2} u_{1} \Leftrightarrow \frac{d^{2} u_{1}}{d x^{2}}=J\left(\lambda^{2} u_{1}\right),
$$

which is equivalent to

$$
\left\{\begin{array}{l}
u_{1} \in H_{0}^{1}(\Omega) \cap H^{2}(\Omega), \\
\frac{d^{2} u_{1}}{d x^{2}}=m \lambda^{2}\left(u_{1}-\left(e_{0} a\right)^{2} \frac{d^{2} u_{1}}{d x^{2}}\right) .
\end{array}\right.
$$

We want to find all solutions of (13). Therefore, we first obtain a set of solutions. It is easily seen that $\varphi_{n}=\sin \left(\frac{n \pi x}{l}\right)$ lies in $H^{2}(\Omega) \cap H_{0}^{1}(\Omega)$. Furthermore, it satisfies (13) if and only if $\lambda_{n}$ satisfies

$$
\lambda_{n}^{2}=-\frac{\left(\frac{n \pi}{l}\right)^{2}}{m\left(1+\left(e_{0} a\right)^{2}\left(\frac{n \pi}{l}\right)^{2}\right)} .
$$

The solution of above equation is denoted as follows

$$
\begin{gathered}
\lambda_{+n}=\sqrt{\frac{\left(\frac{n \pi}{l}\right)^{2}}{m\left(1+\left(e_{0} a\right)^{2}\left(\frac{n \pi}{l}\right)^{2}\right)}} i, \\
\lambda_{-n}=-\sqrt{\frac{\left(\frac{n \pi}{l}\right)^{2}}{m\left(1+\left(e_{0} a\right)^{2}\left(\frac{n \pi}{l}\right)^{2}\right)}} i .
\end{gathered}
$$


For $\lambda_{ \pm n}$ defined by (15) and (16), it is easy to see that

$$
\varphi_{ \pm n}(x)=\left(\begin{array}{c}
\sin \left(\frac{n \pi x}{l}\right) \\
\lambda_{ \pm n} \sin \left(\frac{n \pi x}{l}\right)
\end{array}\right)
$$

lies in the domain of $A$, and satisfies $A \varphi_{ \pm n}=\lambda_{ \pm n} \varphi_{ \pm n}$. Hence, $\varphi_{ \pm n}$ is an eigenfunction of $A$.

Furthermore,

$$
\begin{aligned}
\left\langle\varphi_{-n}, \varphi_{+n}\right\rangle_{e}=\left\|\varphi_{-n}\right\|^{2} & =\left\|\varphi_{+n}\right\|^{2}= \\
& \frac{m l}{4}\left[\left(e_{0} a\right)^{2}\left(\frac{n \pi}{l}\right)^{4}+m\left(\frac{n \pi}{l}\right)^{2}+m\left(e_{0} a\right)^{2}\left(\frac{n \pi}{l}\right)^{4}\right] .
\end{aligned}
$$

Lemma 3. The normalized set of eigenfunctions $\left\{\frac{\varphi_{+n}}{\left\|\varphi_{+}\right\|}, \frac{\varphi_{-n}}{\left\|\varphi_{-n}\right\|}, n \in \mathbb{N}\right\}$ forms a Riesz basis of $\mathcal{H}$.

Proof. It is well-known that $\left\{\frac{1}{\sqrt{\mu_{n}}} \sin \left(\frac{n \pi x}{l}\right), n \in \mathbb{N}\right\}$, with

$$
\mu_{n}=\frac{l}{2}\left(\frac{n \pi}{l}\right)^{4}
$$

forms an orthonormal basis of $H^{2}(\Omega)$.

Let $w=\left(\begin{array}{c}w_{1} \\ w_{2}\end{array}\right) \in \mathcal{H}$. By the above, there exist $\left\{c_{1, n}\right\}_{n \in \mathbb{N}}$ and $\left\{c_{2, n}\right\}_{n \in \mathbb{N}}$ in $\ell^{2}$ such that

$$
\begin{aligned}
& w_{1}(x)=\sum_{n=1}^{\infty} c_{1, n} \frac{1}{\sqrt{\mu_{n}}} \sin \left(\frac{n \pi x}{l}\right), \\
& w_{2}(x)=\sum_{n=1}^{\infty} c_{2, n} \frac{1}{\sqrt{\mu_{n}}} \sin \left(\frac{n \pi x}{l}\right) .
\end{aligned}
$$

Using the normalized eigenfunctions, (20), (21) can be written in the following form

$$
w=\sum_{n=1}^{\infty} d_{+n} \frac{\varphi_{+n}}{\left\|\varphi_{+n}\right\|}+d_{-n} \frac{\varphi_{-n}}{\left\|\varphi_{-n}\right\|}
$$

with

$$
\left\{\begin{array}{l}
\frac{d_{+n}}{\left\|\varphi_{+n}\right\|}+\frac{d_{-n}}{\left\|\varphi_{-n}\right\|}=\frac{c_{1, n}}{\sqrt{\mu_{n}}} \\
\lambda_{+n} \frac{d_{+n}}{\left\|\varphi_{+n}\right\|}+\lambda_{-n} \frac{d_{-n}}{\left\|\varphi_{-n}\right\|}=\frac{c_{2, n}}{\sqrt{\mu_{n}}} .
\end{array}\right.
$$


We write (23) in a matrix notation as follows

$$
\left(\begin{array}{l}
c_{1, n} \\
c_{2, n}
\end{array}\right)=\left(\begin{array}{cc}
\frac{\sqrt{\mu_{n}}}{\left\|\varphi_{+n}\right\|} & \frac{\sqrt{\mu_{n}}}{\left\|\varphi_{-n}\right\|} \\
\frac{\sqrt{\mu_{n}} \lambda_{+n}}{\left\|\varphi_{+n}\right\|} & \frac{\sqrt{\mu_{n}} \lambda_{-n}}{\left\|\varphi_{-n}\right\|}
\end{array}\right)\left(\begin{array}{l}
d_{+n} \\
d_{-n}
\end{array}\right) .
$$

The set $\left\{\frac{\varphi_{+n}}{\left\|\varphi_{+n}\right\|}, \frac{\varphi_{-n}}{\left\|\varphi_{-n}\right\|}, n \in \mathbb{N}\right\}$ forms a Riesz basis of $\mathcal{H}$ if and only if $\left\{d_{ \pm n}\right\}_{n} \in \ell^{2}$ whenever $\left\{c_{ \pm n}\right\}_{n} \in \ell^{2}$. This holds if and only if the matrix in (24) is (uniformly) bounded and (uniformly) boundedly invertible. Since $\lambda_{+n} \neq \lambda_{-n}$, we have that for all $n$ the matrix is invertible. Using (15), (16), (18), and (19), it is easily seen that the coefficients and determinant of the matrix in (24) are (uniformly) bounded and away from zero, which implies that the matrix is uniformly bounded and boundedly invertible.

Since the normalized eigenfunctions $\left\{\frac{\varphi_{+n}}{\left\|\varphi_{+n}\right\|}, \frac{\varphi_{-n}}{\left\|\varphi_{-n}\right\|}, n \in \mathbb{N}\right\}$ form a Riesz basis of $\mathcal{H}$, we have that they are all the eigenfunctions. Using (15) and (16), it is clear that eigenvalues of $A$ are simple, totally disconnected and satisfy (10). This finishes the proof.

Corollary 4. Relations (15) and (16) show that eigenvalues of $A$ have zero real part. Thus, axial vibration of a nanorod is stable phenomena but not exponentially stable.

Knowing that the $A$ is Riesz-spectral operator, it is easy to derive the formula for the $C_{0}$-semigroup, and thus for the exact solution of (7).

\section{Approximate Controllability}

Consider the PDE (1) with initial and homogeneous boundary conditions as defined in equations (2)-(5). We assume that the shaping function $b(x)$ around the control point $x_{0}$ is as follows

$$
b(x)=\frac{1}{2 \epsilon} \chi_{\left[x_{0}-\epsilon, x_{0}+\epsilon\right]},
$$

for some $\epsilon>0$, where $\chi_{J}$ denotes the characteristic function of a set $J$. If $\epsilon$ be close to zero, which we may assume, then $b(x)$ is approximation of Dirac delta distribution around $x_{0}$. This form is used in practical engineering applications $[10$, $14]$.

Theorem 5. Consider the system (1)-(5), where $b(x)$ is defined by (25). The system is approximately controllable if and only if the following condition is satisfied: 


$$
\sin \left(\frac{n \pi x_{0}}{l}\right) \sin \left(\frac{n \pi \epsilon}{l}\right) \neq 0, \quad \forall n \in \mathbb{N}
$$

Proof. Consider the abstract formulation of system (1)-(5) as defined in (7). Using [3] Theorem 4.2.3, shows that (7) is approximately controllable if and only if for all $n \in \mathbb{N}$

$$
\int_{x_{0}-\epsilon}^{x_{0}+\epsilon} \sin \left(\frac{n \pi x}{l}\right) \neq 0 .
$$

The proof is now completed by direct calculation of integral (27).

\section{Conclusion}

The axial vibration of the nanorod is formulated as a first order differential equation. The closed analytical form solution of this equation is obtained by using semigroup theory. It is proved that the axial vibration of the nanorod is stable phenomena but not exponentially stable. Furthermore, it is proved that the axial vibration of the nanorod is approximately controllable under an algebraic condition.

\section{References}

[1] M. Aydogdu, Axial vibration of the nanorods with the nonlocal continuum rod model, Physica E, 41 (2009), 861-864.

[2] Q. Chen, C. Udomsangpetch, S.C. Shen, Y.C. Liu, Z. Chen and X.T. Zeng, The effect of $\mathrm{AlOOH}$ boehmite nanorods on mechanical property of hybrid composite coatings, Thin Solid Films, 517 (2009), 4871-4874.

[3] R.F. Curtain and H. Zwart, An Introduction to Infinite-Dimensional Linear Systems Theory, Springer-Verlag, New York, 1995.

[4] A.C. Eringen, Nonlocal Continuum Field Theories, Springer-Verlag, New York, 2002.

[5] A.C. Eringen, On differential equations of nonlocal elasticity and solutions of screw dislocation and surface waves, J. Appl. Phys., 54 (1983), 47034710. 
[6] V.A. Fonoberov and A.A. Balandin, Low-frequency vibrational modes of viruses used for nanoelectronic self-assemblies, Phys. Stat. Sol., 12 (2004), R67-R69.

[7] S. Guillon, D. Saya, L. Mazenq, S. Perisanu, P. Vincent, A. Lazarus, O. Thomas and L. Nicu, Effect of non-ideal clamping shape on the resonance frequencies of silicon nanocantilevers, Nanotechnology, 22 (2011), 245501.

[8] H. Heidari, H. Zwart and A. Malek, Analysis of the three dimensional heat conduction model in nano- or micro-scale, Proc. of the 19th International Symposium on Mathematical Theory of Networks and Systems (MTNS) (2010), 1877-1882.

[9] G. Heinrich and F. Marquardt, Coupled multimode optomechanics in the microwave regime, Europhys. Lett., 93 (2011), 18003.

[10] H. Ichimura and P. Todd, Implementing Nonparametric and Semiparametric Estimators, Handbook of Econometrics, Elsevier Science, 2007.

[11] A.Y. Kasumov, I.I. Khodos, P.M. Ajayan and C. Colliex, Electrical resistance of a single carbon nanotube, Europhys. Lett., 34 (1996), 429-434.

[12] Z. Ounaies, C. Park, K.E. Wise, E.J. Siochi and J.S. Harrison, Electrical properties of single wall carbon nanotube reinforced polyimide composites, Composites Science and Technology, 63 (2003), 1637-1646.

[13] S. Narendar and S. Gopalakrishnan, Nonlocal scale effects on ultrasonic wave characteristics of nanorods, Physica E, 42 (2010), 1601-1604.

[14] J.J. Winkin, D. Dochain and P. Ligarius, Dynamical analysis of distributed parameter tubular reactors, Automatica, 36 (2000), 349-361.

[15] G. Xu and D. Feng, The Riesz basis property of a Timoshenko beam with boundary feedback and application, IMA J. App. Math., 67 (2002), 357370. 